\title{
The design of a high-temperature MEMS pressure sensor with integrated temperature compensation and signal-conditioning circuits
}

\author{
Zong Yao, ${ }^{1,2, a}$, Wang-Wang Li ${ }^{1,2, b}$, Di-Ya Zhang ${ }^{1,2, c}$, \\ Lei Qi ${ }^{1,2, \mathrm{~d}}$, Bin Zhang ${ }^{1,2, \mathrm{e}}$, Cheng Lei ${ }^{1,2, \mathrm{f}}, \mathrm{Xin} \mathrm{Li}^{1,2, \mathrm{~g}}$, \\ Ting Liang ${ }^{1,2, \dagger}$ and Ji-Jun Xiong ${ }^{1,2, \mathrm{~h}}$ \\ ${ }^{I}$ National Key Laboratory for Electronic Measurement Technology, \\ North University of China, Taiyuan 030051, China; \\ ${ }^{2}$ Key Laboratory for Instrumentation Science \& Dynamic Measurement, \\ North University of China, Ministry of Education, Taiyuan 030051, China; \\ ayaozong126@sina.com; ${ }^{b} 18434365707 @ 163 . c o m ;$ \\ c18234157832@163.com; 'dilei19850224@163.com; \\ ezb0003@126.com; fleichengnuc@163.com; $2496136118 @ q q . c o m ;$

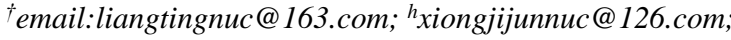

\begin{abstract}
The design of a high-temperature MEMS pressure sensor with integrated temperature compensation and signal-conditioning circuits is introduced in the paper, including the design and fabrication of pressure-sensitive chip, the temperature compensation method, the signal-conditioning circuit and the sensor's assembly structure. According to the test result, the sensor has the advantages of high accuracy and sensitivity, low temperature drift and $0 \mathrm{~V} \sim 5 \mathrm{~V}$ standard voltage signal output. The range of the sensor working temperature is from $-50{ }^{\circ} \mathrm{C}$ to $220{ }^{\circ} \mathrm{C}$, which is suitable for use in high temperature environments
\end{abstract}

Keywords: High-temperature piezoresistive pressure sensor; Temperature compensation; Signal-conditioning circuit

\section{Introduction}

Nowadays, the high-temperature MEMS pressure sensors based on SOI (silicon on insulate) material are widely used in the fields of petrochemicals, energy and electric power, and aerospace [1,2]. However, the major defects of the sensors are parameters drift in wide temperature range and RFI (radio frequency interference) on the signal transmission link, which seriously reduces their measurement accuracy. The parameters drift on offset voltage and output sensitivity is caused by the increased piezoresistance and decreased piezoresistive coefficient with temperature[4]; and RFI is due to small output voltage ranges in the dozens to hundreds of millivolts susceptible to ambient noise[5]. 
In this work, the developed sensors exhibit less temperature drift and stronger anti-interference performance due to a $0 \mathrm{~V}$ to $5 \mathrm{~V}$ standard voltage signal output. The measurement accuracy of the sensor is significantly improved and expected to be highly applicable for pressure measurements in harsh environments with large temperature variations.

\section{Fabrication}

\subsection{Design and fabrication of the pressure-sensitive chip}

The testing principle of the pressure-sensitive chip is Wheatstone bridge, as shown in Fig.1(a). The stress distribution of pressure sensitive diaphragm is shown in Fig.1 (b). The piezoresistors should be placed at midpoints of the four sides in the diaphragm for stress concentration, in order to improve the sensitivity of the sensor, as shown in Fig.1(c,d).

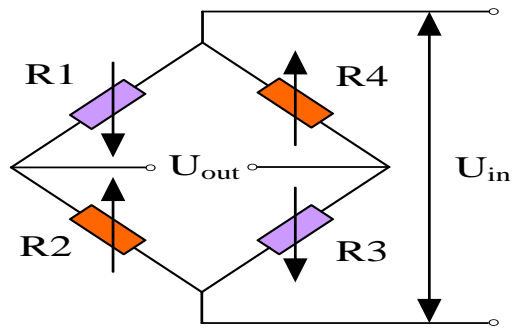

(a) Wheatstone bridge circuit diagram;

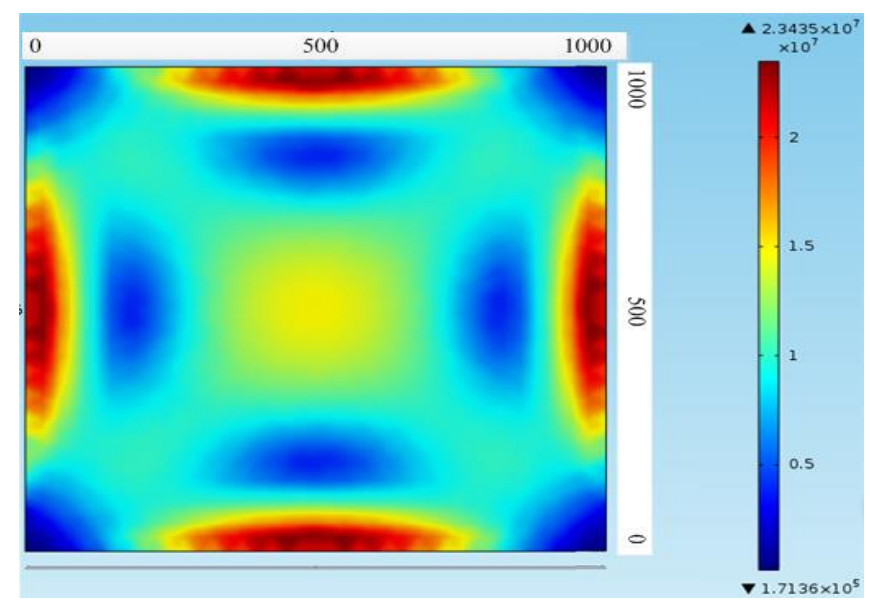

(b) stress distribution in diagram; 


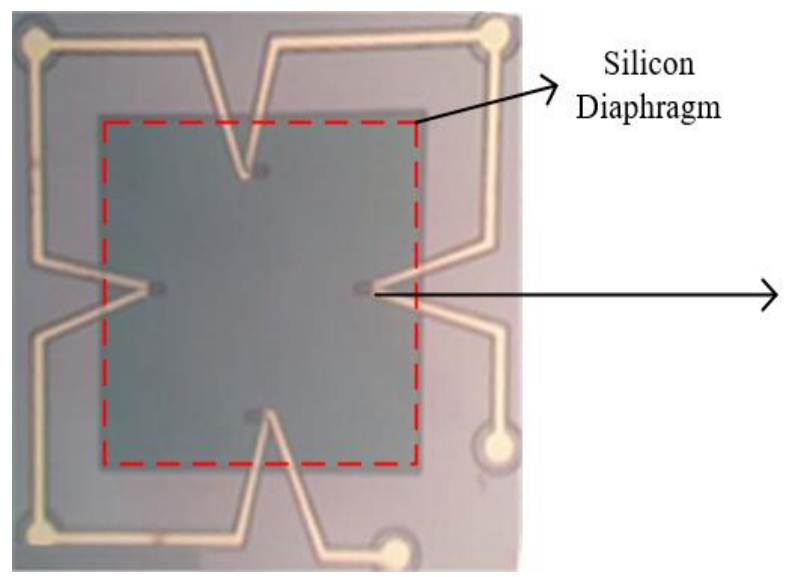

(c) plan view of the chip;

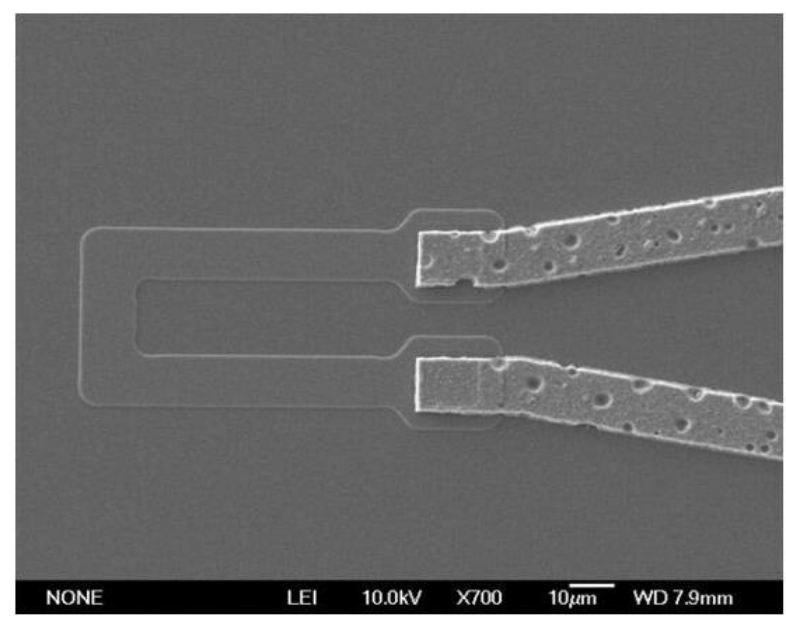

(d) enlarged view of the piezoresistor

Figure 1. The pressure-sensitive chip's equivalent circuit and structure:

a square shape is application in sensitive membrane, as shown in Figure 1(c). Generally, a sensitive silicon diaphragm of thickness $h$ with side length a should meet the following three requirements: first, output linearity requires that the sensitive diaphragm should follow the small deflection theory model, which means that the maximum deflection of the diaphragm is less than $20 \%$ of the thickness of the center[6]; second, the maximum overload stress requires that the combined stress value (the difference of longitudinal stress and transverse stress) at each point on the film is less than or equal to $30 \%$ of the material rupture stress[7]; third, the higher sensitivity output requires that the relative rate of 
change of the varistor be greater than $2 \%$ [8]. According to the shell theory formula [9], the requirements above can be restated by Equation (1):

The square design is used in pressure sensitive diaphragm shape, as shown in Figure 1(c). In general, the size of the diaphragm thickness ' $h$ ' and side length 'a' should meet the three constraints:

1) The output linearity requires that the elastic membrane should meet the requirements of the small deflection theory model, which means that the maximum deflection on the membrane center is less than $20 \%$ of the thickness ' $h$ ' $[6]$;

2) Pressure overload derating design requires that the maximum overload stress on the film is less than $30 \%$ of the material rupture stress[7];

3) High sensitivity output requires that the variation of the varistor should be greater than $2 \%$ under the full scale pressure loading[8].

According to the shell theory formula [9], the requirements above can be restated by Equation (1):

$$
\left\{\begin{array}{l}
w_{\max }=0.01518 \frac{p a^{4}\left(1-v^{2}\right)}{E h^{2}} \leq 20 \% h \\
\max \left(\left|\sigma_{1}-\sigma_{t}\right|\right)=0.308 \mathrm{P}_{\max } \frac{\mathrm{a}^{2}}{\mathrm{~h}^{2}}(1-v) \leq 0.3 \sigma_{m} \\
\frac{\Delta R}{R}=\frac{0.308}{2} \pi_{44} P \frac{a^{2}}{h^{2}}(1-v) \geq 2 \%
\end{array}\right.
$$

The developed high temperature pressure sensor sensitive chip is shown in Fig. 2(a). The manufacturing processes is shown in Fig. 2(b), including several steps: (a) SOI wafer; (b) Boron doping; (c) Etching piezoresistors; (d) Depositing a passivation layer; (e) Etching the ohmic contact region; (f) Heavy doping in lead area for ohmic contact; (g) Sputtering metal lead; (h) deeply etching back cavity for the pressure-sensitive diaphragm; (i) anodic bonding for the absolute reference chamber.

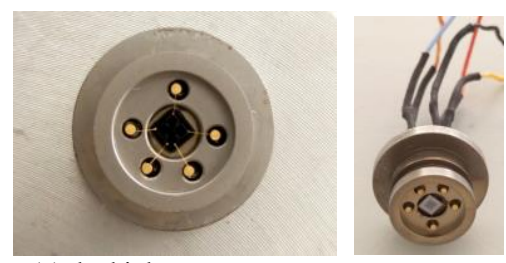

(a) the high-temperature pressure sensor; 

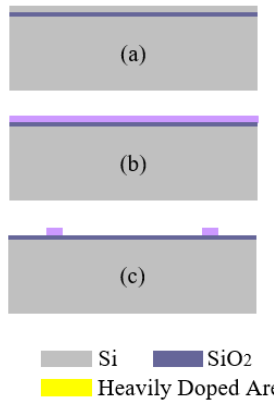

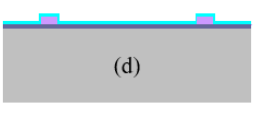

(e)

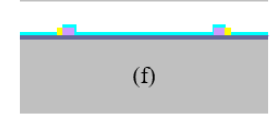

Device Layer Metal Lead b) the MEMS fabrication process

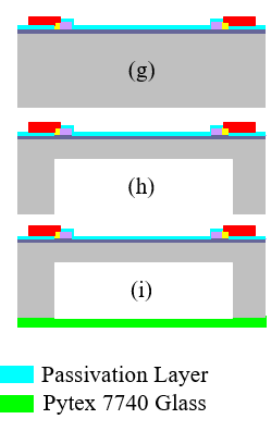

Figure 2. The developed high-temperature pressure sensor and fabrication process;

Compared to traditional manufacturing processes, a novel design in device area sink is used to avoid damage to resistances, metal leads and sensitive diaphragms in anodic bonding process, as shown in Fig.3. In the case of anodic bonding, the cathode plate in contact with the silicon wafer is not directly pressed against the resistor strip due to the device area sink, which reduces the packaging residual stress and improves product quality and reliability.

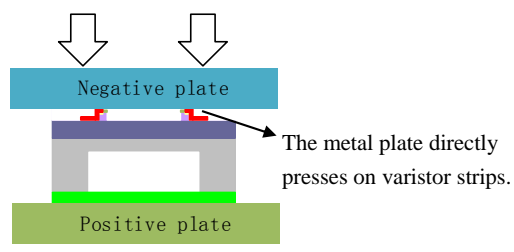

(a) a traditional manufacturing processes in anodic bonding ;

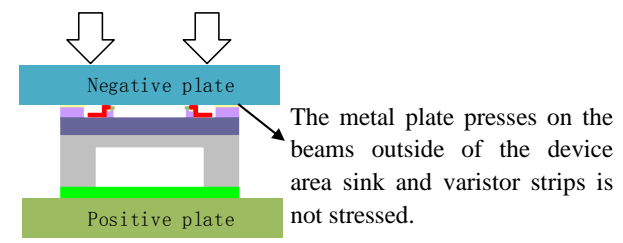

(b) a device area sink in anodic bonding.

Figure 3. Comparison of anodic bonding model;

\subsection{Temperature compensation}

The proposed passive resistor temperature compensation models for the sensor powered by constant voltage supply are shown in Fig.4, and their output voltage expression of which are shown in Equation (2) and (3). 


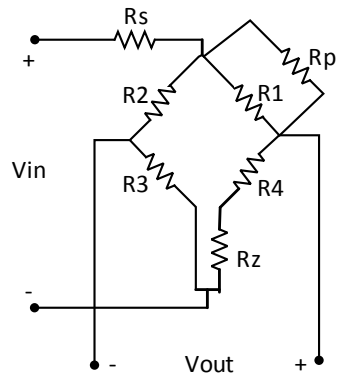

(a)

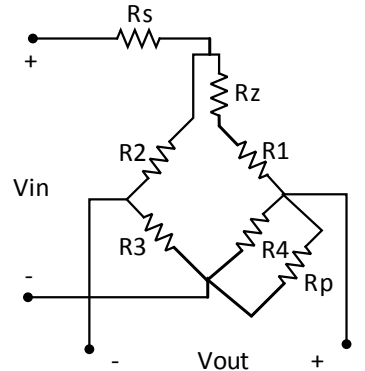

(b)

Figure 4. Passive resistor temperature compensation model with a constant voltage supply: (a) compensation model for negative initial offset voltage; (b) compensation model for positive initial offset voltage.

$$
\begin{aligned}
& V_{\text {OUT }}(T, P)=V_{I N} \times \frac{\left[R_{2}(T, P)+R_{3}(T, P)\right] \|\left[R_{Z}+R_{4}(T, P)+R_{1}(T, P) \| R_{P}\right]}{\left[R_{2}(T, P)+R_{3}(T, P)\right] \|\left[R_{Z}+R_{4}(T, P)+R_{1}(T, P) \| R_{P}\right]+R_{S}} \times \\
& {\left[\frac{R_{Z}+R_{4}(T, P)}{R_{Z}+R_{4}(T, P)+R_{1}(T, P) \| R_{P}}-\frac{R_{3}(T, P)}{R_{2}(T, P)+R_{3}(T, P)}\right]}
\end{aligned}
$$$$
\mathrm{V}_{\text {oUT }}(\mathrm{T}, \mathrm{P})=\mathrm{V}_{\text {IN }} \times \frac{\left[\mathrm{R}_{2}(\mathrm{~T}, \mathrm{P})+\mathrm{R}_{3}(\mathrm{~T}, \mathrm{P})\right] \|\left[\mathrm{R}_{\mathrm{Z}}+\mathrm{R}_{1}(\mathrm{~T}, \mathrm{P})+\mathrm{R}_{4}(\mathrm{~T}, \mathrm{P}) \| \mathrm{R}_{\mathrm{P}}\right]}{\left[\mathrm{R}_{2}(\mathrm{~T}, \mathrm{P})+\mathrm{R}_{3}(\mathrm{~T}, \mathrm{P})\right] \|\left[\mathrm{R}_{\mathrm{Z}}+\mathrm{R}_{1}(\mathrm{~T}, \mathrm{P})+\mathrm{R}_{4}(\mathrm{~T}, \mathrm{P}) \| \mathrm{R}_{\mathrm{P}}\right]+\mathrm{R}_{\mathrm{S}}} \times
$$$$
\left[\frac{R_{4}(T, P) \| R_{P}}{R_{Z}+R_{1}(T, P)+R_{4}(T, P) \| R_{P}}-\frac{R_{3}(T, P)}{R_{2}(T, P)+R_{3}(T, P)}\right]
$$

Where $\mathrm{R} 1(\mathrm{~T}, \mathrm{P})$ to $\mathrm{R} 4(\mathrm{~T}, \mathrm{P})$ are bridge arm resistance value under the temperature $\mathrm{T}$ and pressure $\mathrm{P} ; \mathrm{Rz}, \mathrm{Rp}, \mathrm{Rs}$ are low temperature coefficient compensation resistance which is temperature independent; symbol 'I' represents resistance in parallel.

According to the uncompensated sensor's initial offset voltage, we can select the compensation model in Fig. 4(a) or Fig. 4(b) and the corresponding computational formula. The model parameters needed to test in advance involve the four bridge arm resistances at the temperature thresholds T0, T1 (T0< T1) and the load pressures P0, P1 (P0< P1). These values are presented in Table 1.

Table 1. Measurement parameters for compensation model

\begin{tabular}{ccccc}
\hline & R1 & R2 & R3 & R4 \\
\hline (T0, P0) & R1(T0, P0) & R2(T0, P0) & R3(T0, P0) & R4(T0, P0) \\
(T0, P1) & R1(T0, P1) & R2(T0, P1) & R3(T0, P1) & R4(T0, P1) \\
(T1, P0) & R1(T1, P0) & R2(T1, P0) & R3(T1, P0) & R4(T1, P0) \\
(T1, P1) & R1(T1, P1) & R2(T1, P1) & R3(T1, P1) & R4(T1, P1) \\
\hline
\end{tabular}


The algorithm equations for passive resistor temperature compensation are listed as:

$$
\left\{\begin{array}{l}
\mathrm{V}_{\text {OUT }}\left(\mathrm{T}_{0}, \mathrm{P}_{0}\right)=\mathrm{U}_{0} \\
\frac{\partial \mathrm{V}_{\mathrm{OUT}}\left(\mathrm{T}, \mathrm{P}_{0}\right)}{\partial \mathrm{T}}=0 \\
\frac{\partial \mathrm{V}_{\mathrm{OUT}}\left(\mathrm{T}, \mathrm{P}_{1}\right)}{\partial \mathrm{T}}=0
\end{array}\right.
$$

The temperature compensation requires that the temperature coefficient of offset and sensitivity are independent of temperature. This means that the partial derivatives of $\operatorname{Vout}(\mathrm{T}, \mathrm{P} 0)$ and $\operatorname{Vout}(\mathrm{T}, \mathrm{P} 1)$ with respect to temperature $\mathrm{T}$ must remain equal to zero.The Compensation model parameter values of $\mathrm{Rz}, \mathrm{Rp}$, Rs can be calculated by solving the Eq. (4) using MATLAB[10].

\subsection{Signal-conditioning}

A high-temperature signal-conditioning circuit is used to amplify the sensor output voltage signal from tens of millivolts to $0 \mathrm{~V} \sim 5 \mathrm{~V}$ in order to improve sensitivity and reduce RFI. A high temperature operational amplifier (HT1104) and a linear regulator (HTPLREG05) produced by Honeywell (Morris Plains, NJ, the USA) are used, as shown in Fig. 5.

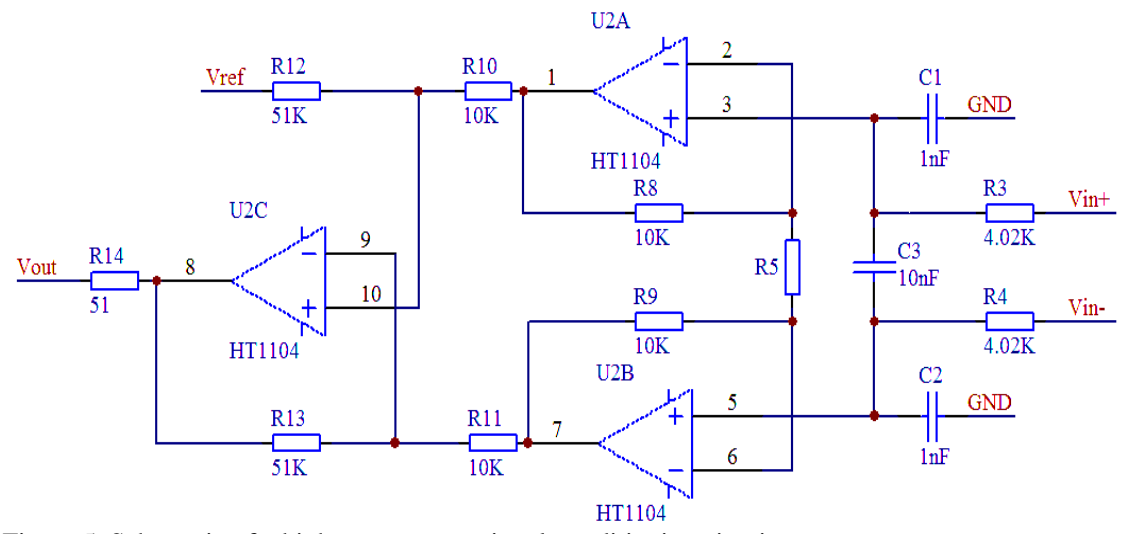

Figure 5. Schematic of a high-temperature signal-conditioning circuit

Where, Vin+ and Vin- are the output voltages of the bridge, Vref is the output zero reference voltage of the circuit, and R5 determines the output voltage range of the high-temperature signal-conditioning circuit, which can be expressed as: 


$$
\mathrm{V}_{\text {OUT }}=5.1 \times\left(1+\frac{20 \mathrm{~K}}{\mathrm{R} 5}\right) \times\left(\mathrm{V}_{\mathrm{in}+}-\mathrm{V}_{\mathrm{in}-}\right)+\mathrm{V}_{\text {ref }}
$$

\subsection{Sensor structure}

The assembly structure and pictures of the high-temperature piezoresistive pressure sensor with integrated signal-conditioning circuit are shown in Figures 6. The device has the characteristics of small size, light weight, easy dismantling, and good sealing performance.

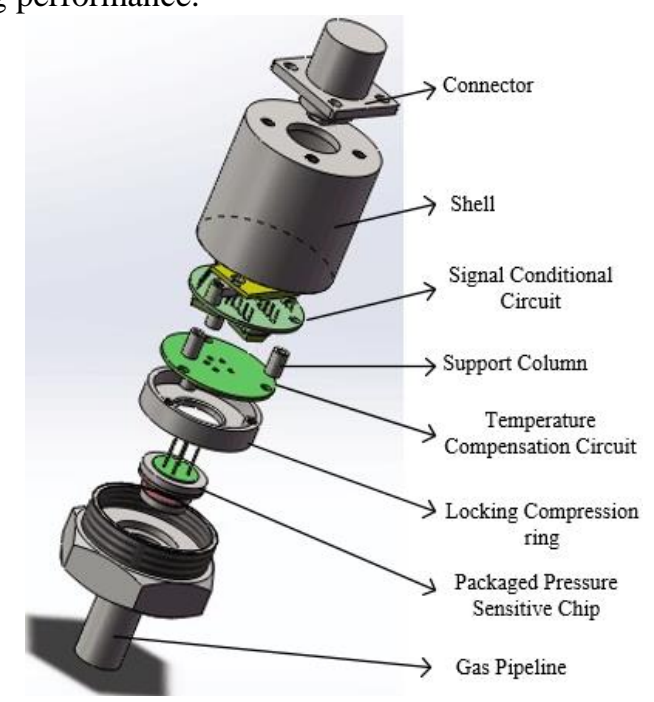

Figure 6. Sensor structure
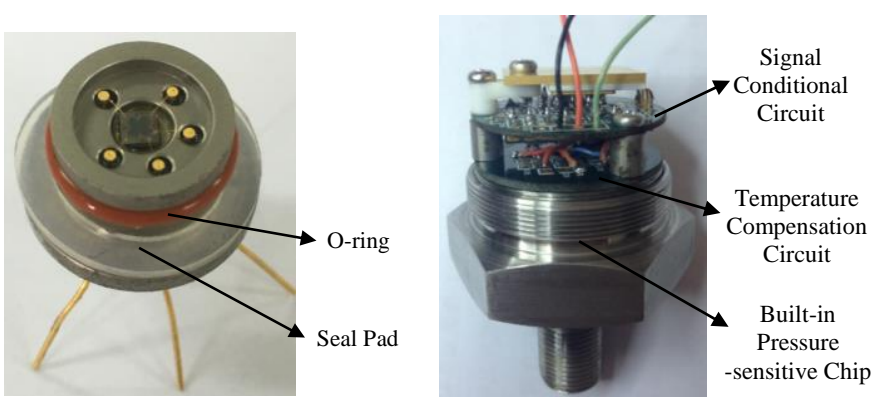


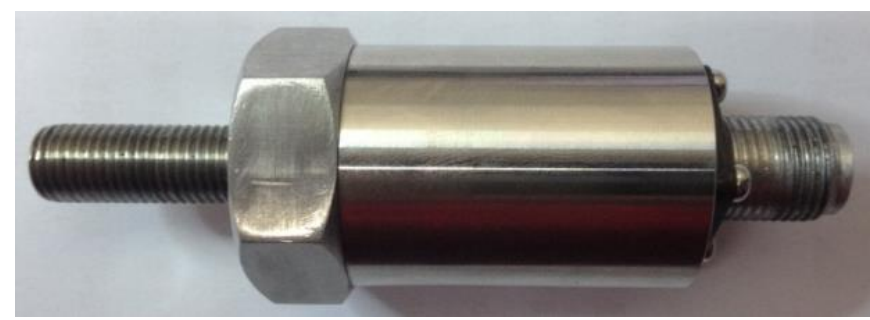

I. Performance Test

The high-temperature pressure sensor can be tested using the calibration device shown in Fig. 7 in the range $20 \sim 220^{\circ} \mathrm{C}$ and $100 \sim 2000 \mathrm{kPa}$

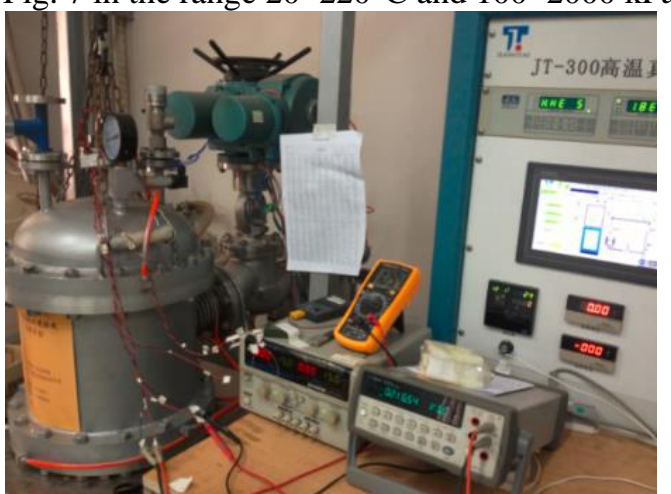

Figure 7. High-temperature and pressure calibration device developed by the authors

Test results for the uncompensated high-temperature pressure sensor is shown in Fig.8(a), where the sensor's initial offset voltage is negative and the output voltage decreases significantly as the temperature increases. Test results for the sensor with integrated temperature compensation is shown in Fig.8(b), where the sensor's initial offset voltage is positive and temperature drift is greatly reduced. Final test results for the sensor with integrated temperature compensation and signal-conditioning circuits is shown in Fig.8(c), where the output voltage range of the sensor is $0 \sim 5 \mathrm{~V}$, the sensitivity is $210 \mathrm{mV} / 100 \mathrm{kPa}$ and the total accuracy overall the working temperature is $\pm 1.5 \% \mathrm{FS}$ 


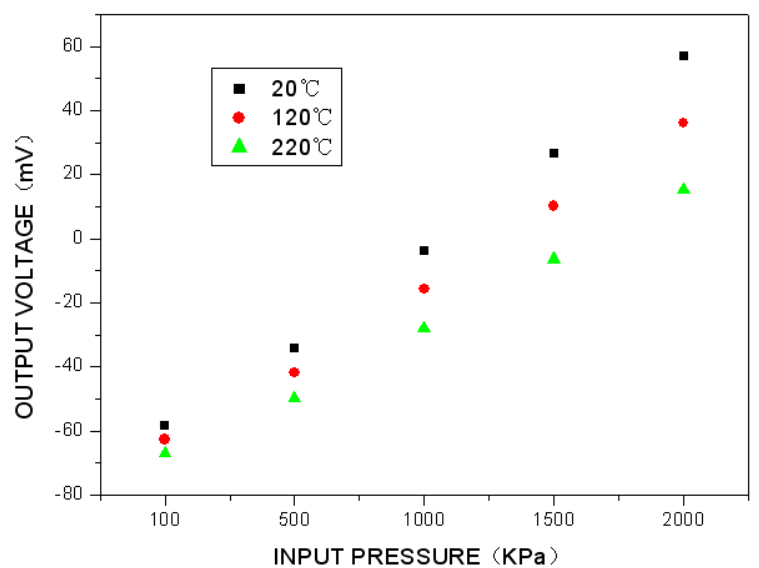

(a) output voltage calibration curve for the uncompensated sensor;

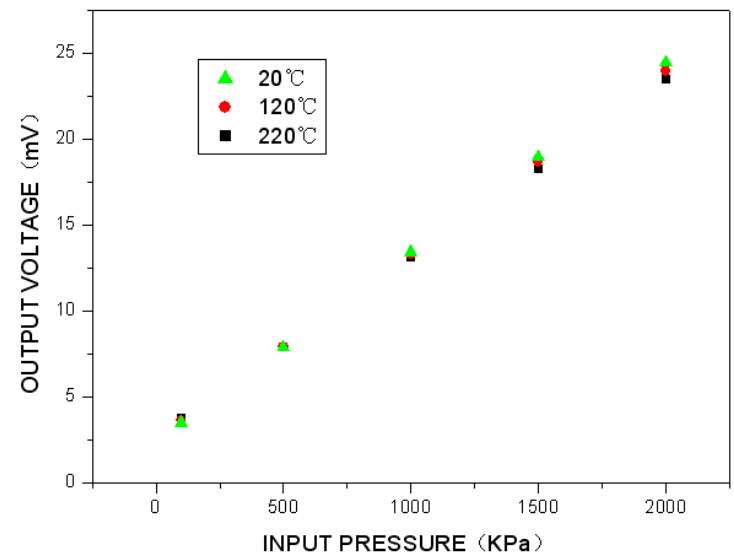

(b) output voltage calibration curve for temperature compensation; 


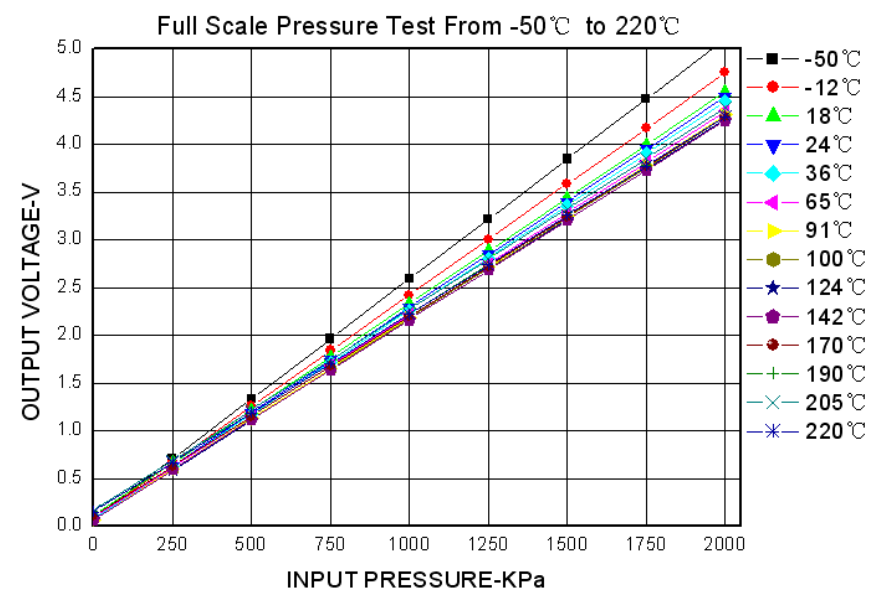

(c) output voltage calibration curve for temperature compensation and signal-conditioning circuits Figure 8. Pressure sensor calibration test results:

\section{Conclusions}

In this paper, we have presented the design of a high-temperature MEMS pressure sensor with integrated temperature compensation and signal-conditioning circuits. It can be seen from the final result that the sensor has features with high precision from $20^{\circ} \mathrm{C}$ to $220^{\circ} \mathrm{C}$, great sensitivity $210 \mathrm{mV} / 100 \mathrm{kPa}$ and $0 \mathrm{~V} \sim 5 \mathrm{~V}$ standard voltage signal output. It is expected that the performance of the sensor can be further improved by optimizing design specifications and process parameters, so that it can be capable of meeting more applications in harsh environments with large temperature variations.

\section{Acknowledgements}

This work was supported by National Natural Science Foundation of China (51405454).

\section{References}

[1] Shuwen Guo, Harald Eriksen, Kimiko Childress, High temperature smart-cut SOI pressure sensor, Sensors and Actuators A:Physical[J], (2009)255-260.

[2] M.Aryafar, M.Hamedi, M.M.Ganjeh, A novel temperature compensated piezoresistive pressure sensor, Measurement[J], (2015)25-29.

[3] Feng Wen, Fang Niu, Wendong Zhang, Tao Guo, The analyses of MEMS pressure sensor temperature drift and the design of compensated circuit, Journal of Projectiles,Rockets,Missiles and Guidance[J], (2006)862-865. 
[4] C. Pramanik, T. Islam, H. Saha, Temperature compensation of piezoresistive micro-machined porous silicon pressure sensor by ANN, Microelectronics Reliability[J], (2006)343-351.

[5] Guanwu Zhou, Yulong Zhao, Fangfang Guo, Wenju Xu, A Smart High Accuracy Silicon Piezoresistive Pressure Sensor Temperature Compensation System, Sensors[J], (2014)12174-12190.

[6] Quan Wang, Jianning Ding, Wenxiang Wang, Fabracation and temperature coefficient compensation technology of low cost high temperature pressure sensor, Sensors and Actuators A:Physical[J], (2005)468-473.

[7] M. H. Bao, Micro Mechanical Transducers-Pressure Sensors, Accelerometers and Gyroscopes, Dutch Amsterdam Publisher, (2000) pp.257-262.

[8] Singh J, Rajanna K, Reddy A M, et al. Effect of Process Deviations on Performance of Piezoresistive Pressure Sensors[J]. IEEE Transactions on Semiconductor Manufacturing, 2014, 27(3):410 - 416.

[9] V.Mosser, J.Suski, J.Goss, E. Obermeier, Piezoresistive pressure sensors based on polycrystalline silicon, Sen.Actuators, A28(1991)113-132.

[10] Singh S S, Dinakrushna M. Solution of the reactor point kinetics equations by MATLAB computing[J]. Nuclear Technology \& Radiation Protection, 2015, 30(1):11-17. 